\title{
Nonlinear Schrödinger equation with chaotic, random, and nonperiodic nonlinearity
}

\author{
W. B. Cardoso, ${ }^{1}$ S. A. Leão, ${ }^{1}$ A. T. Avelar, ${ }^{1}$ D. Bazeia, ${ }^{2}$ and M. S. Hussein ${ }^{3}$ \\ ${ }^{I}$ Instituto de Física, Universidade Federal de Goiás, 74001-970, Goiânia, GO, Brazil. \\ ${ }^{2}$ Departamento de Física, Universidade Federal da Paraíba, 58051-970, João Pessoa, PB, Brazil. \\ ${ }^{3}$ Departamento de Física Matemática, Instituto de Física, \\ Universidade de São Paulo, 05314-970, São Paulo, SP, Brazil
}

\begin{abstract}
In this paper we deal with a nonlinear Schrödinger equation with chaotic, random, and nonperiodic cubic nonlinearity. Our goal is to study the soliton evolution, with the strength of the nonlinearity perturbed in the space and time coordinates and to check its robustness under these conditions. Comparing with a real system, the perturbation can be related to, e.g., impurities in crystalline structures, or coupling to a thermal reservoir which, on the average, enhances the nonlinearity. We also discuss the relevance of such random perturbations to the dynamics of Bose-Eisntein Condensates and their collective excitations and transport.

PACS numbers: 42.65.Tg; 42.25.Dd; 05.45.Pq
\end{abstract}

The nonlinear Schrödinger equation (NLSE) is the mathematical vehicle that describes the evolution of solitonic solutions for different nonlinear systems, such as, fiber optics [1], bulk medium and photonic crystals [2], Langmuir waves in plasmas [3], wave function of Bose-Einstein condensates (BECs) [4], and others.

A special case involving the NLSE consists in variable coefficients modulated in the spatial and/or temporal coordinates. The control of these coefficients allow us to obtain new distinct solutions. In this context, [5, 6] have recently proposed a treatment of BECs using similarity transformations to construct explicit nontrivial solutions of the cubic and cubicquintic NLSE with potentials and nonlinearities depending both on time and on the spatial coordinates. Also, thermal effects on nonlinearities can change its form, presenting new solutions [7]. In BECs, the $s$-wave scattering length of interatomic collisions determines the strength of the nonlinearity coefficient [4], and it can be controlled using the Feshbach resonance (FR) [8] via external magnetic [9, 10] or optical [11] fields. The FR mechanism allows for a practical means to manipulate the nonlinearity [12].

Although the control of the nonlinearity has been very effective, noises can appear in the system management or added to it. In this way, the perturbations can change the nonlinearity, and thus influencing possible changes in the noise-free solutions. The inclusion of spatial random potential in the study of BEC dynamics has been proposed in Refs. [13, 14, 15, 16] and recently was tested experimentally [17]. When randomness is introduced in the BEC dynamics, through an optical speckle, one may be able to study Anderson localization in the context of BEC and superfluidity [13]. It was clearly demonstrated in these papers that in the presence of disorder the condensate's expansion in 1D waveguides is inhibited, and the collective dipole and quadrupole oscillations are strongly damped. In a way, this is similar to the damping of collective states in nuclei and metal clusters [18], where the randomness is internal.

A natural question arises as to what would be the consequence of having the disorder present directly in the nonlinearity term? For BEC, this implies a point two-body inter- action (t-matrix) with a random component. Would this add or remove some of the effects of the speckle potential? In this connection, [19] have recently proposed an NLSE in the presence of random nonlinearity. Specifically, these authors consider the effects of random time modulation of the nonlinearity coefficient on the dynamics of solitary waves in the NLSE. On the other hand, to our knowledge, chaotic perturbations in the nonlinearity term in the NLSE have not been fully considered yet, though it is a common knowledge that several physical systems do exhibit chaotic behavior.

Classically chaotic systems appear to behave as random systems. Tiny differences in the initial state of the system can lead to enormous differences in the final state even over fairly small time scales [20]. This happens even though these systems are deterministic, meaning that their future dynamics are fully determined by their initial conditions with no random elements involved. This behavior is known as deterministic chaos.

The dynamical systems theory (DST) is an area whose interest lies mainly in nonlinear phenomena, the source of classical chaos. DST groups use several concepts to the study of chaos, such as Lyapunov exponents, fractal dimension, bifurcation, and symbolic dynamics among other elements [20]. Recently, other approaches have been considered, such as information dynamics and entropic chaos degree [21]. For example, given $C_{0}, C_{n}$ can be the $n$-th iterate of the quadratic functions: $C_{n}(\mu)=C_{n-1}^{2}+\mu$; sine functions: $C_{n}(\mu)=$ $\mu \sin \left(C_{n}-1\right)$; logistic functions $C_{n}(\mu)=\mu C_{n-1}\left(1-C_{n-1}\right)$; exponential functions: $C_{n}(\mu)=\mu \exp \left(C_{n-1}\right)$; doubling function defined on the interval $[0,1): C_{n}=2 C_{n-1} \bmod 1$, and so on, $\mu$ being a parameter. It is worth recalling that all the functions in the above list are familiar to researchers in DST. For example, for some values of $\mu$, it is known that some of these functions can behave in quite a chaotic manner [20]. In what follows we make a distinction between chaos and randomness, though both concepts indicate disorder.

The major thrust of our paper is to verify the influence of the different types of perturbations in the nonlinearity of a system governed by NLSE. In this sense, we investigate the chaotic, random, and nonperiodic nonlinearity perturbation. We know 
that these perturbations are different, however, to what extent the overall effect is universal (independent on the details of the random perturbations), and how can it modify a solitonic solution? We purports to supply some answers to the above.

Differently from Ref. [19], which considers a random time modulation on a certain point (generating a Gaussian distribution), here we consider a constant background nonlinearity perturbed by a random function that interferes in both spatial and temporal coordinates. Surprisingly, some of the solutions found here can move depending of the amount of perturbed points in the nonlinearity. This fact is similar to those studied by the thermal effects on the nonlinearity [7].

Firstly we consider the NLSE given by

$$
i \psi_{t}=-\psi_{x x}+g(x, t)|\psi|^{2} \psi
$$

where $\psi=\psi(x, t), x$, and $t$ dimensionless, and $g(x, t)$ is the function that describe the nonlinearity of the system. Here we consider

$$
g(x, t)=G(1+\sigma(x, t))
$$

where $G$ and $G \sigma(x, t)$ are the nonlinear parameter and the coefficient generated by a chaotic, random, or nonperiodic generator, respectively. Eq. (1) describes, e.g., the density of particles in a Bose-Einstein condensates when it is free of external potentials, in a configuration type cigar-shaped; the spatial pulse propagation in bulk crystals that present Kerr-effect in the nonlinearity or temporal pulse propagation in nonlinear optical fiber; etc.

We investigate the evolution of the solution for the NLSE via numerical simulations, based on the split-step finite differences (SSFD) method with a time-step and space-step size of $\Delta t=0.0001$ and $\Delta x=0.01$, respectively; we use $N$ to represent the number of points in space. The core of SSFD is based on the Crank-Nicolson algorithm [22]. To control these numerical simulations we looked for the conserved quantity given by (power)

$$
P=\sum_{x=1}^{N}|\psi(x, t)|^{2}
$$

To calculate the error we use the comparative form

$$
E_{r}=\frac{1}{N} \sum_{x=1}^{N}\left(|\psi(x, 0)|^{2}-\left|\psi\left(x, t_{f}\right)\right|^{2}\right)
$$

where $t_{f}$ is the final time of the evolution. The equation above represents a mean distance between the input and output state.

When we take $G=-2$ and $\sigma(x, t)=0$ in (2), we can write

$$
\psi=e^{i \mu t} \operatorname{sech}(x)
$$

as solution of (1), with $\mu=1$. This solution will be taken as initial condition for our simulations of the Eq. (1).

For our numerical simulations we consider the chaotic perturbation in the nonlinearity given by the logistic function, the
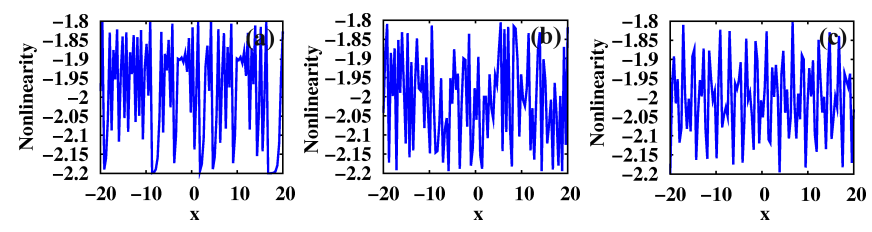

FIG. 1: Plots of the perturbed nonlinearity for the (a) chaotic, (b) random, (c) and nonperiodic functions.

random perturbation is generated by random algorithm simulator, and the nonperiodic perturbations are generated by the function $\alpha(\cos (5 x) / 2+\cos (\sqrt{5} x) / 2)$, where $\alpha$ assumes the values of the perturbation. In Figs. 1a, 1b, and 1 we wisplay a form of the chaotic, random, nonperiodic nonlinearity as functions of space for a generic time, respectively. Experimentally, this perturbation in the nonlinearity can be constructed, e.g., in a crystal with impurities which are altered chaotically, randomly, or non-periodically. It remains to be seen what the presence of $G \sigma(x, t)$ implies, though one would guess that it amounts to taking into account, within the mean field, GrossPitaevskii, description, the effects of the many-body correlations.

We use (5) as input state in (1) to verify its evolution in the presence of the above mentioned perturbations. In Fig. 2a we consider the chaotic perturbation via $\sigma(x, t)$ between \pm 0.001 and \pm 0.1 (10\%). The chaotic perturbation is obtained considering 4000 points affected within the interval $-20 \leq x \leq 20$ in space, that changes the spatial profile of the nonlinearity. These perturbed points are changed by a new function after a time $t=20$, and so we will have 100 temporal points affected in the interval $0 \leq t \leq 200$. Fig. 2 b displays the soliton amplitude (height) of the solution $\left(|\psi|^{2}\right)$ at the position $x=0$. Note that it becomes vanishingly small after $t=60$ owing to its motion. However, the soliton is stable in this range of perturbation. We calculated the error in the power of $2.41 \times 10^{-4}$ and the comparative error of $E_{r}=9.39$.

On the other hand, when we consider a chaotic perturbation of $50 \%$ in the value of the amplitude, we found that the soliton practically disappears. This case is shown in Fig. 3. In the Figs. 3a and 3 b we display the $|\psi|^{2}$ and the height in the position $x=0$, respectively. The error in the power was $2.38 \times 10^{-4}$ with a mean distance $E_{r}=9.76$. In this case the mean distance is due to the moving and the vanishing pattern, i.e., the output state can not be at the same position as that of the input state.

Now, when we consider a random perturbation of the nonlinearity, obtained here by an algorithm of random number generation, we note a different behavior of the solutions. Here the soliton remains stable even for $50 \%$ of perturbation, differently from the case of the chaotic perturbation. In Fig. 4 a we plot the soliton solution considering $10 \%$ of random perturbation in the nonlinearity using the same arguments presented for the chaotic case, i.e., 4000 affected points in space versus 100 temporal points into the range shown. Fig. 4b displays the amplitude of the soliton at $x=0$. This perturbation is 

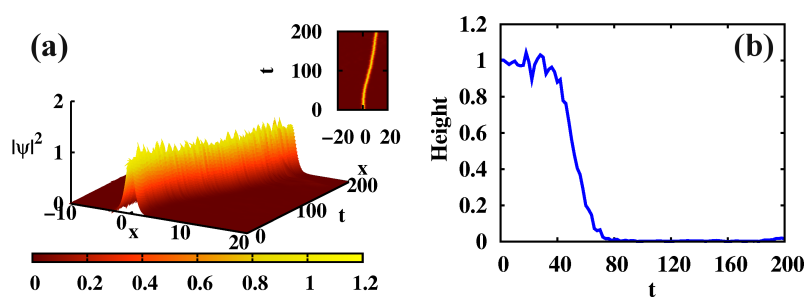

FIG. 2: Plots of the soliton evolution $|\psi(x, t)|^{2}$ with $10 \%$ of chaotic perturbation in the nonlinearity. In (a) is displayed the solution and its profile (top panel) and (b) its height at position $x=0$.
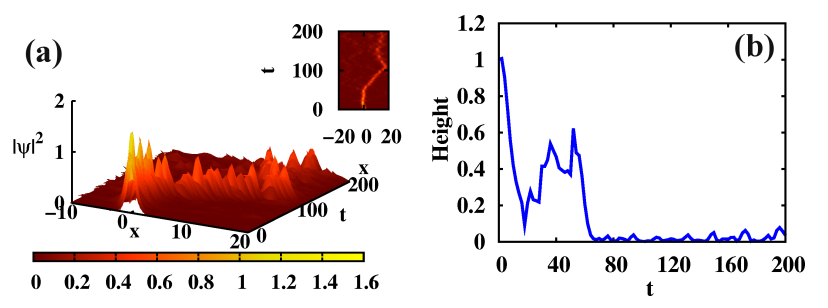

FIG. 3: Plots of the soliton evolution $|\psi(x, t)|^{2}$ with $50 \%$ of chaotic perturbation in the nonlinearity (see text for details). In (a) is displayed the solution and its profile (top panel) and (b) its height at position $x=0$.

responsible for moving the soliton. The error in the power is of $4.88 \times 10^{-6}$ with the mean distance between the input and output states of $10^{-1}$. For $50 \%$ of random perturbation the oscillation of the soliton is more evidenced when compared with the case of $10 \%$ of random perturbation. Figs. 5 and 5 b show the $|\psi|^{2}$ and the amplitude for $x=0$, respectively. The robustness is guaranteed, differently form the chaotic case. The errors in power and the mean distance are $1.17 \times 10^{-5}$ and $10^{-1}$, respectively.

To conclude our study we investigate the effects of the nonperiodic perturbation in the nonlinearity. From Fig. 1t one observes that this perturbation seems the most well-behaved compared to the other two types. This fact is reflected in the behavior of the solution which remains practically with the same form as that of the input state. This occurs even when it suffers $50 \%$ of nonperiodic perturbation, as can be seen in Fig. 6. The error in the power is of $2.83 \times 10^{-4}$ and the mean distance is of 2.88 .

With the results presented here, we verify that, under similar conditions, chaotic, random, and nonperiodic perturbations in the nonlinearity can present distinct features, and sometimes results in vanishing solitons, as verified when the system suffers chaotic perturbations.

In summary, in the present work we have studied the effects of chaotic, random, and nonperiodic perturbations in the nonlinearity on the soliton evolution via NLSE. We considered cubic nonlinearity with strength perturbed chaotically, randomly, or nonperiodically. In the chaotic case we found that moving solitons can be destroyed when they are perturbed with $50 \%$ in the intensity of the nonlinearity. On the other hand, when the system engenders random perturbation this
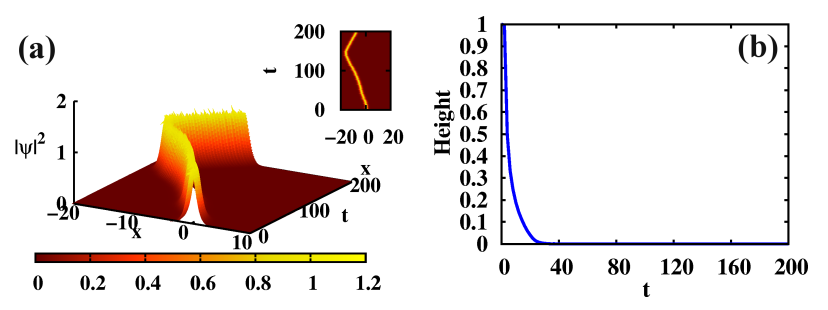

FIG. 4: Plots of the soliton evolution $|\psi(x, t)|^{2}$ with $10 \%$ of random perturbation in the nonlinearity (see text for details). In (a) is displayed the solution and its profile (top panel) and (b) its height at position $x=0$.
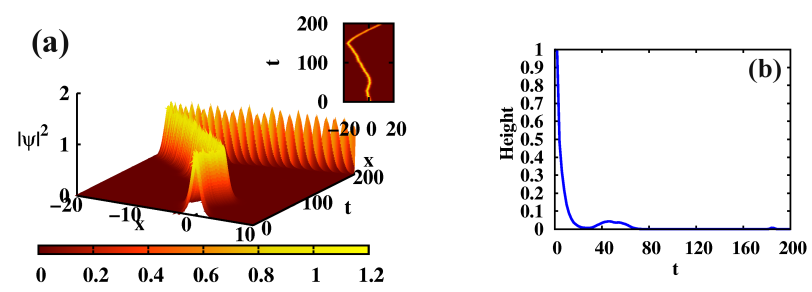

FIG. 5: Plots of the soliton evolution $|\psi(x, t)|^{2}$ with $50 \%$ of random perturbation in the nonlinearity (see text for details). In (a) is displayed the solution and its profile (top panel) and (b) its height at position $x=0$.

does not occur. The soliton solution remains stable, however now it can move. Finally, when we look for the nonperiodic perturbation we found that it displays robust solutions with no apparent influence on the solitons. In a way, we can say that disorder in the non-linearity may, or may not lead to Anderson-type localization, depending on the nature of the perturbation. Our results have direct impact on work in optical lattices with impurities in the crystal, laser-generated randomness in the non-linearity, and many-body effects in the dynamics of Bose-Einstein condensates and their collective excitations and transport.

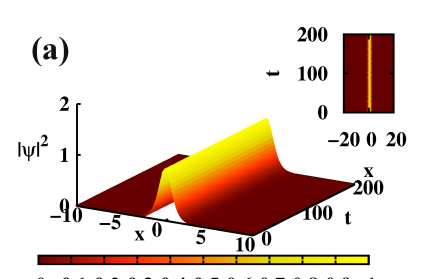

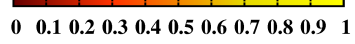

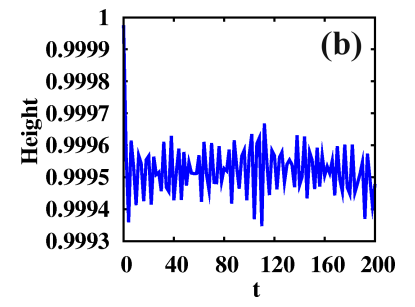

FIG. 6: Plots of the soliton evolution $|\psi(x, t)|^{2}$ with $50 \%$ of nonperiodic perturbation in the nonlinearity (see text for details). In (a) is displayed the solution and its profile (top panel) and (b) its height at position $x=0$. 


\section{Acknowledgments}

The authors would like to thank CAPES, CNPQ, FUNAPE$\mathrm{GO}$, and FAPESP for partial financial support.

[1] G. P. Agrawal, Nonlinear Fiber Optics (Academic Press, San Diego, 2001).

[2] Y. S. Kivshar and G. P. Agrawal, Optical Solitons: From Fibers to Photonic Crystals (Academic Press, San Diego, 2003).

[3] V. E. Zakharov, Sov. Phys. JETP 35, 908 (1972).

[4] C. J. Pethick and H. Smith, Bose-Einstein Condensation in Dilute Gases (Cambridge Univ. Press, Cambridge, 2002); L. P. Pitaevskii and S. Stringari, Bose-Einstein Condensation (Oxford Univ. Press, Oxford, 2003).

[5] J. Belmonte-Beitia, V. M. Pérez-García, V. Vekslerchik, and V. V. Konotop, Phys. Rev. Lett. 100, 164102 (2008).

[6] A. T. Avelar, D. Bazeia, and W. B. Cardoso, Phys. Rev. E 79, 025602(R) (2009).

[7] A. Gharaati, P. Elahi, and S. Cari, Acta Phys. Pol. A 112, 891 (2007); M. A. Molchan, E. V. Doktorov, and R. A. Vlasov, J. Opt. A: Pure Appl. Opt. 11, 015706 (2009).

[8] For an extensive review see, e.g., E. Timmermans, P. Tommasini, M. Hussein and A. Kerman, Phys. Reports, 315, 199 (1999).

[9] T. Köhler, K. Goral, and P. S. Julienne, Rev. Mod. Phys. 78, 1311 (2006).

[10] S. Inouye, M. R. Andrews, J. Stenger, H. J. Miesner, D. M. Stamper-Kurn, and W. Ketterle, Nature 392, 151 (1998); J. Stenger, S. Inouye, M. R. Andrews, H. J. Miesner, D. M. Stamper-Kurn, and W. Ketterle, Phys. Rev. Lett. 82, 2422 (1999); J. L. Roberts, N. R. Claussen, J. P. Burke Jr., C. H.
Greene, E. A. Cornell, and C. E. Wieman, Phys. Rev. Lett. 81, 5109 (1998); S. L. Cornish, N. R. Claussen, J. L. Roberts, E. A. Cornell, and C. E. Wieman, Phys. Rev. Lett. 85, 1795 (2000).

[11] F. K. Fatemi, K. M. Jones, and P. D. Lett, Phys. Rev. Lett. 85, 4462 (2000); M. Theis, G. Thalhammer, K. Winkler, M. Hellwig, G. Ruff, R. Grimm, and J.H. Denschlag, Phys. Rev. Lett. 93, 123001 (2004).

[12] B. A. Malomed, Soliton Management in Periodic Systems (Springer, New York, 2006).

[13] J. E. Lye, L. Fallani, M. Modugno, D. Wiersma, C. Fort, and M. Inguscio, Phys. Rev. Lett. 95, 070401 (2005); C. Fort, L. Fallani, V. Guarrera, J. Lye, M. Modugno, D. S. Wiersma, and M. Inguscio, Phys. Rev. Lett. 95, 170410 (2005); L. Fallani, J. E. Lye, V. Guarrera, C. Fort, and M. Inguscio, Phys. Rev. Lett. 98, 130404 (2007).

[14] Y. P. Chen, J. Hitchcock, D. Dries, M. Junker, C. Welford, R. G. Hulet, Phys. Rev. A 77, 033632 (2008).

[15] F. K. Abdullaev and J. Garnier, Phys. Rev. A 72, 061605 (2005).

[16] E. Akkermans, S. Ghosh, and Z.H. Musslimani, J. Phys. B: At. Mol. Opt. Phys. 41, 045302 (2008).

[17] T. Schwartz, G. Bartal, S. Fishman, and M. Segev, Nature 446, 52 (2007); Y. Lahini, A. Avidan, F. Pozzi, M. Sorel, R. Morandotti, D. N. Christodoulides, and Y. Silberberg, Phys. Rev. Lett. 100, 013906 (2008).

[18] See, e.g., M. S. Hussein, V. Kharchenko, L. F. Canto and R. Donangelo, Ann, Phys. (N. Y.) 284, 167 (2000).

[19] Q. Y. Chen, P. G. Kevrekidis, and B. A. Malomed, Phys. Lett. A 373, 1361 (2009).

[20] R. L. Devaney, An Introduction to Chaotic Dynamical Systems (Westview Press, 1989).

[21] M. Ohya, Int. J. Theor. Phys. 37, 495 (1998).

[22] F. J. Vesely, Computational Physics: An introduction (Plenum Press, New York, 1994). 